\title{
A Comparative Study on the Different Staining Methods and Number of Specimens for the Detection of Acid Fast Bacilli
}

\author{
Mustafa Ulukanligil ${ }^{+}$, Gonul Aslan, Sami Tasçi
}

Department of Microbiology, Harran University Medical School, Sanliurfa, Turkey

The presence of acid fast bacilli in multiple specimens was investigated comparatively with ZiehlNeelsen $(Z N)$ and fluorescence microscopy $(F M)$ staining in order to determine sensitivity in detecting tuberculosis (TB). A total of 465 specimens obtained from 295 patients were analysed at Harran University Medical School Hospital between March 1998 and March 2000. The culture was employed as the reference method. Sixty-eight patients (23.1\%) were diagnosed as having TB by culture. The ZN and FM staining sensitivities were $67.6 \%$ (46/68) and $85.2 \%$ (58/68) respectively. Two hundred and one patients (68.1\%) submitted one specimen to the laboratory. TB positivity was detected in $42(20.9 \%)$ of these patients by culture. The sensitivities of ZN and FM stains were found to be $61 \%$ and $83 \%$ in these patients. However, in 18 patients $(6.1 \%)$ who submitted two specimens to the laboratory, the TB was positive in six of them (33.3\%) and ZN and FM sensitivities were $66 \%$ and $83 \%$ respectively. When three specimens or more were collected from the patients (76 patients, 25.8\%), TB positivity was determined in 20 of them (26.3\%) and the sensitivities were $80 \%$ and $92 \%$ in the ZN- and FM-stained smears, respectively.

Our data indicate that in the diagnosis of TB, FM has greater sensitivity than ZN. In particular, in the case of a single specimen, the diagnostic value of FM is quite significant. It is, therefore, possible to conclude that both ZN and FM staining can be used for the diagnosis of TB when there are more than two specimens. However, if only one or two specimens are available, FM staining is preferable.

Key words: Mycobacterium tuberculosis - sensitivity - Ziehl Neelsen - fluorescence microscopy

Tuberculosis (TB) remains a major health problem, with an estimate 8 million new cases and 3 million deaths due to this disease every year around the world (Dixie et al.1993). Three hundred to four hundred new cases are reported every year in Sanliurfa, Turkey out of a population 400,000 (S.urfa tuberculosis dispensary statistics report, 1998). Several factors are responsible for the outbreak of TB in the region: the deterioration of socioeconomic conditions, with low income and inadequate education, and great ignorance of the disease, as shown by the fact that patients often stop taking medicine when their symptoms have disappeared. Although human immunodeficiency virus is the major factor in TB outbreaks in places like the United States and Africa, it is quite rare in southern Turkey.

Most standard laboratory textbooks (Janda 1997, Sommers et al. 1988) and guidelines for mycobacteriology laboratories (Kent \& Kubica

\footnotetext{
${ }^{+}$Corresponding author. Fax: +90-414-3151180. E-mail: mulukan@bigfoot.com

Received 27 January 2000

Accepted 12 July 2000
}

1985) suggest that at least three specimens, preferably collected on three consecutive days, should be submitted to the laboratory for acid-fast bacilli (AFB) smear and culture by patients suspected of having tuberculosis. The detection of AFB in direct smears prepared with concentrated sputa, urine and specimens of other body fluids has considerable clinical and epidemiological value and remains the most widely used rapid diagnostic test for TB in most developing countries (Nelson et al. 1998). While new radiometric and molecular diagnostic techniques have been developed and are widely used in the developed world (Heifets 1997), it is estimated that between $60 \%$ and $70 \%$ of all TB cases are diagnosed by means of sputum smear examination (Guthie et al. 1993). Two traditional staining methods, namely fluorescence microscopy (FM) and the Ziehl-Neelsen (ZN) method are readily available (Nagpaul 1967). The ZN method has commonly been used around the world, particularly in developing countries, because of its simplicity and low cost (Guthie et al. 1993). Several reports (Lempert 1944, Boyd \& Marr 1975, Janda 1997) have indicated that FM has a greater sensitivity and saves time when compared to the $\mathrm{ZN}$ method. One disadvantage of the FM technique is that it may sometimes yield false positive results. However, most of these can be prevented by 
restaining the smear according to the $\mathrm{ZN}$ method for bright-light microscopy (Holst et al. 1959, Heifets 1997).

In the present study, these two techniques were compared in terms of sensitivity in the diagnosis of TB, and the correlation between sensitivity and the number of specimens collected was examined. In addition, in the light of our results and available literature, we tried to identify those techniques suitable to our circumstances.

\section{MATERIALS AND METHODS}

Two hundred and ninety-five patient suspected of having TB were referred to our clinic (Clinical Microbiology Laboratory, Harran University Medical School Hospital) and a total of 460 specimens were collected from them between March 1998 and March 2000. These specimens consisted of 430 sputum, 17 urine, 4 gastric lavage and 3 cervical abscess samples, 3 pleural fluids, 1 peritoneal fluid, 1 cervical lymph node biopsy specimen and 1 semen specimen.

All the sputum samples were decontaminated and concentrated using the N Acetyl-L-CysteineSodium Hydroxide procedure recommended by the Center of Disease Control and Prevention (Kent \& Kubica 1985). The sputum, urine and other specimens were prepared using the conventional centrifugation method and the smears were stained with carbolfuchsin (ZN) and Auramin-O (fluorochrome) stains (Ebersole 1995). The sputum, urine and other specimen sediments were inoculated onto Lowenstein-Jensen media and incubated at $37^{\circ} \mathrm{C}$ in $5 \% \mathrm{CO}_{2}$ for up to six weeks (Lambi 1995). The culture was used as the reference method and compared with the $\mathrm{ZN}$ and FM techniques. In recording the AFB numbers determined in the stained smears under immersion microscopy (x1000 magnification), the following reporting scale was used: more than 9 AFB per field (4+), 1-9 AFB per field (3+), 1-9 AFB per 10 fields (2+), 1-9 AFB per 100 fields (1+). When only a few AFB were detected in 300 fields, the results were considered questionable. In order to equate the number of bacilli observed with FM (400x magnification) to the number of bacilli observed with immersion microscopy (1000x magnification), the values were divided by 4 (American Thoracic Society 1981).

\section{RESULTS}

Sixty-eight of the 295 (23.1\%) patients were diagnosed as having Mycobacterium tuberculosis by the isolation of the organism from cultures of sputum (66 cases), abscess ( 1 case) and lymph node ( 1 case) samples. Forty-six patients (15.5\%) tested positive with ZN staining while $58(19.6 \%)$ tested positive with FM staining (Table I). Hence, sensitivity of FM staining was $86 \%$ and that of the $\mathrm{ZN}$ staining was $67 \%(\mathrm{p}<0.05)$.

Two hundred-one patients $(68.1 \%)$ submitted one specimen for AFB examination and in 42 (20.9\%), the organism was isolated from sputum (40 cases), abscess material (1 case) and lymph node biopsy material ( 1 case). In these 42 patients, 28 sputum samples yielded positive results with ZN staining and 38 (37 sputum, 1 cervical abscess) yielded positive results with FM staining. The sensitivities of the $\mathrm{ZN}$ and FM staining were $61 \%$ and $83 \%$ respectively $(\mathrm{p}<0.05)$ (Table II).

We observed that smears which were positive with both $\mathrm{ZN}$ and FM contained between 1 to 9 AFB per fields. The 14 positive smears, which were not detected with ZN-stained smears, were detected low-density bacilli (1-2 bacilli per 100 fields) on FM-stained smears. Only one false positive result was seen with FM method, hence, specificities of the $\mathrm{ZN}$ and FM staining were found as $100 \%$ and $99 \%$ respectively.

Eighteen $(6.1 \%)$ patients submitted two specimens to the laboratory and in $33.3 \%$ (6 out of 18) of these patients, the organism was recovered in 1 or 2 of the sputum samples. Four of these produced positive results with ZN staining (sensitivity, 66\%) and 5 produced positive results with FM staining

TABLE I

Evaluation of two different stains and culture results for patients submitting to one, two and three specimens

\begin{tabular}{lccccccc}
\hline & \multicolumn{5}{c}{ Number of patient (\%) } \\
\cline { 2 - 6 } No. of & \multicolumn{2}{c}{ Ziehl-Neelsen staining } & Fluorochrome staining & \multicolumn{2}{c}{ Culture } & \multirow{2}{*}{ Total (\%) } \\
\cline { 2 - 6 } specimen & Positive (\%) & Negative (\%) & Positive (\%) & Negative (\%) & Positive (\%) & Negative (\%) & \\
\hline 1 & $26(12.9)$ & $175(87.1)$ & $36(17.9)$ & $165(82.1)$ & $42(20.9)$ & $159(79.1)$ & $201(68.1)$ \\
$2^{a}$ & $4(22.2)$ & $14(77.8)$ & $5(27.8)$ & $13(72.2)$ & $6(33.3)$ & $12(66.7)$ & $18(6.1)$ \\
$3^{a}$ & $16(21.1)$ & $60(78.9)$ & $18(23.7)$ & $58(76.3)$ & $20(26.3)$ & $56(73.7)$ & $76(25.8)$ \\
\hline Total & $46(15.5)$ & $249(84.5)$ & $58(19.6)$ & $237(80.3)$ & $68(23.1)$ & $227(76.9)$ & $295(100)$
\end{tabular}

$a:$ at least one specimen was positive. 
TABLE II

Sensitivity, specificity, positive and negative predictive values of two differently stained smears on the basis of culture positivities for patients submitting one, two and three specimens for acid-fast bacilli detection

\begin{tabular}{lllllll}
\hline & \multicolumn{3}{c}{ Ziehl-Neelsen staining } & \multicolumn{3}{c}{ Fluorescence microscopy staining } \\
\hline No. of specimen & 1 & 2 & 3 & 1 & 2 & 3 \\
Sensitivity & 0.61 & 0.66 & 0.80 & 0.83 & 0.83 & 0.92 \\
Specificity & 1 & 1 & 1 & 0.99 & 1 & 1 \\
+ predictive value & 1 & 1 & 1 & 0.97 & 1 & 1 \\
- predictive value & 0.90 & 0.85 & 0.93 & 0.95 & 0.92 & 0.96 \\
\hline
\end{tabular}

(sensitivity, 83\%). Two positive patients were not detected on the basis of $\mathrm{ZN}$-stained smears. The smears of one patient, which were stained with MF, contained 3-5 bacilli in every 30-40 fields while the smears of the other patient were negative with both FM and ZN. No false positive result was detected in these patients.

Three or more specimens were submitted by $25.8 \%$ of the patients (76 out of 153) and 20 of these patients (26.3\%) were diagnosed as having TB by isolating the organism in one or more of the sputum specimens. Sixteen of them produced positive results with $\mathrm{ZN}$ staining (sensitivity, 80\%) and 18 produced positive results with FM staining (sensitivity, $92 \%$ ). No false positive result was detected in these patients (Table II).

Seventeen urine specimens were collected for AFB but none of these were found to be positive in stained smears or cultures. Likewise, other specimens ( 3 pleural fluid, 4 gastric lavage, 1 peritoneal fluid, 2 cervical abscess specimens and 1 semen specimen) were found to be negative with $\mathrm{ZN}$ and FM staining and culture, except for one cervical abscess specimen which yielded positive result with FM staining and culture. One cervical lymph-node specimen yielded $M$. tuberculosis in culture only.

\section{DISCUSSION}

Turkey is a developing country and TB is still a common disease, particularly in the southeast of the county. In Sanliurfa, a city in the southeast, economic conditions are still very poor and 300400 new TB patients are recorded every year.

In recent years, several radiometric and molecular techniques have been developed for the diagnosis of TB. Radiometric techniques reduce the turnover time for AFB. However, they are not suitable for this region of Turkey because of their high cost (Heifets 1997). There is little scope even for using molecular techniques because of our limited financial resources. Conventional methods such as $\mathrm{ZN}$ and FM are rapid, simple techniques with quite low cost. Only ZN has so far been used for the diagnosis of TB in this region. However, it has a low level of sensitivity compared to the FM method. The present study was our first attempt to use FM and compare it with ZN.

We carried out in a comparative study the reliability of the FM and ZN methods in the examination of smears for AFB. We found that the specificities of FM and ZN were $99 \%$ and $100 \%$, and the sensitivities with one sample were $83 \%$ and $61 \%$, respectively. When the number of specimens was increased, the sensitivity of both types of staining increased. When we utilised more than two specimens, the sensitivities were found to be similar. Therefore, it is possible to say that if we have less than three samples, the reliability of FM is much greater than that of $\mathrm{ZN}$. Unless more than one sample was submitted, which was not easy existing socio-economic and cultural conditions, FM proved to be more reliable than the $\mathrm{ZN}$ method. Moreover, another advantage of FM was that it enabled the detection of positive smears, which were overlooked with ZN-stained smears containing low-density bacilli. The use of FM significantly increases the diagnostic value of the smear, particularly where there are low-density bacilli which may escape detection on $\mathrm{ZN}$-stained smears. Our results were in agreement with those of Guthie et al. (1993), Ba and Rieder (1999), and Pollack and Wieman (1977), who reported that FM appears to be more likely to detect TB in smears which contain low-density bacilli.

Many reports (Sommers et al. 1988, Nelson et al. 1998) have shown that mycobacteria may be released irregularly from the lungs, and that it is even possible to find positive and negative results in the same patient during the same period. Thus, it is advised that more than one specimen be collected from patients suspected of having TB. Some other reports (Ozturkeri et al. 1997, Durupinar et al. 1997, Pollock \& Wieman 1997) have claimed that false positive reactions might occur with FM. We observed only one false positive result in present study. Moreover, no false positive reactions occurred in our investigation with the $\mathrm{ZN}$ method. 
Our priority in this study was to find a rapid and cost-effective method for the diagnosis of TB in Sanliurfa. We conclude that the FM method is quite economical in terms of both time and expense and it is recommended for laboratories handling large number of sputum specimens. We discovered that FM is more reliable than $\mathrm{ZN}$. However, all staff who is to examine sputum samples should become familiar with FM in order to prevent false positive and/or negative results. Therefore, it is suggested that until there are qualified staff for the investigation of AFB, the smears should be stained with both of these stains and then examined comparatively. After laboratory staff become familiar with the FM method, this method can be used routinely on its own.

\section{REFERENCES}

American Thoracic Society 1981. Diagnostic standards and classification of tuberculosis and other mycobacteral diseases. Am Rev Respir Dis 123: 343358.

Ba F, Rieder HL 1999. A comparison of fluorescence microscopy with the Ziehl-Neelsen technique in the examination of sputum for acid fast bacilli. Int $J$ Tuberc Lung Dis 3: 1101-1105.

Boyd JR, Marr JJ 1975. Decreasing reliability of acid fast smear techniques of detection of tuberculosis. Ann Intern Med 82: 487.

Dixie E, Snider JR, Dooley WS 1993. Nozocomial tuberculosis in the AIDS era with emphasis on multi drug resistant disease. Heart and Lung 22: 365-369.

Durupýnar B, Birinci A, Gunaydýn M, Sanic A 1997. The reliability of auramine-rhodamine staining technique in the diagnosis of tuberculosis. Bull Microbiol 31: 369-374.

Ebersole LL 1995. Acid-fast stain procedures in mycobacteriology. In HD Isenberg, Clinical Microbiology Procedures Handbook, Amer Soc Microbiol, Washington DC, Section 3.5.

Guthie W, Kitui F, Juma ES, Obvana DO, Mwai J,
Kwamanga D 1993. A comparative study on the reliability of the fluorescence microscopy and ZiehlNeelsen method in the diagnosis of pulmoner tuberculosis. East Afri Med J 70: 263-266.

Heifets L 1997. Mycobacteriology laboratory. Clin Chest Med 18: 35-41.

Holst E, Mitchson DA, Radhakrishna S 1959. Examination of smears for tubercle bacilli by fluorescence microscopy. Ind J Med Res 47: 495.

Janda WM 1997. Mycobacteria. In EW Koneman, SD Allen (eds), Colour Atlas and Textbook of Diagnostic Microbiology, 5 ed., JB Lippincott Co., Philadelphia, p. 893-949.

Kent PT, Kubica GP 1985. Public Health Mycobacteriology: A Guide for Level III Laboratory, US Department of Health and Human Services, CDC, Atlanta, p. 3-10.

Lambi EA 1995. Medium selection and incubation for the isolation of mycobacteria in mycobacteriology. In HD Isenberg, Clinical Microbiology Procedures Handbook, Amer Soc Microbiol, Washington DC, Section 3.6.

Lempert H 1944. Fluorescence microscopy in the detection tubercle bacilli. Lancet 2: 818 .

Nagpaul DR 1967. District tuberculosis control programme in concept and outline. Ind J Tubercle 14: 186

Nelson SM, Deike MA, Cartwright CP 1998. Value of examining multiple sputum specimens in the diagnosis of pulmoner tuberculosis. J Clin Microbiol 36: 467-469.

Ozturkeri H, Demir MA, Balli S, Aydilek R 1997. Comparative evaluation of different staining methods in the diagnosis of tuberculosis. Bull Microbiol 31:231236.

Pollock HM, Wieman EJ 1977. Smear results in the diagnosis of mycobacteriosis using blue light fluorescence microscopy. Clin Microbiol 5: 329-331.

Sommers HM, McClatchy JK, Morello JA 1988. Laboratory Diagnosis of the Mycobacteriosis, CUMITECH 16, Amer Soc Microbiol Washington DC, p. 3-8. 